\title{
An External ac Bias Induced Expansion of Dynamic Voltage Bands in a Weakly Coupled GaAs/AlAs Superlattice
}

\author{
H.T. He ${ }^{1}$, Z.Z. Sun $^{1}$, X.R. Wang ${ }^{1}$, Y.Q. Wang ${ }^{1}$, W.K. Ge ${ }^{1}$, J.N. Wang ${ }^{1, *}$ \\ ${ }^{1}$ Physics Department, Hong Kong University of Science and Technology, Clear Water Bay, Hong Kong, China
}

\begin{abstract}
In intermediate temperature region, an ac induced expansion of dynamic voltage band (DVB) is observed in a weakly coupled GaAs/AlAs superlattice. An analysis in nonlinear terminology suggests that the effect of an external ac signal is to tighten the unstable limit cycle onto the stable fixed point. Beyond certain critical value, the stable limit cycle is the only stable solution of this system.
\end{abstract}

\section{INTRODUCTION}

Since the pioneering work of Esaki and Chang [1], the vertical transport of weakly coupled superlattice (SL) has been shown to exhibit many interesting behaviors. It includes the saw-tooth-like currentvoltage (I-V) characteristics [2], self-sustained current oscillation (SSCO) [3-4] and chaos [5]. It's understood that the formation of stable electric field domain (EFD) in SL is responsible for the occurrence of sawtooth like I-V characteristics and the SSCO is attributed to the traveling of domain boundary within SL [6], which can be induced by varying the doping density $\left(\mathrm{N}_{\mathrm{D}}\right)$ [3], temperature $(\mathrm{T})$ or transverse magnetic field (B) [4]. In 1999, J.N.Wang et.al. [4] pointed out that in the transition from static to dynamic EFD, a so-called dynamic voltage band (DVB) emerged at each saw-tooth like branch. In general, the control parameter spaces $\left(\mathrm{N}_{\mathrm{D}}, \mathrm{T}\right.$ or $\left.\mathrm{B}\right)$ can be divided into three regions. One corresponds to static EFD, in which only saw-tooth like I-V characteristics is observed, the other dynamic EFD, in which the whole sequential tunneling plateau exhibits SSCOs. In between, there's an intermediate region, where alternating DVB and static voltage band (SVB) are observed. In this region the applied dc bias is an additional control parameter for the system stability. In this work, we focus on the influence of an external ac bias on the DVB in the intermediate region of temperature. It is found that the extra ac bias can induce the DVB and increase the width of DVB as the ac amplitude increases. A possible mechanism is given in the term of general nonlinear physics analysis.

\section{EXPERIMENTAL RESULTS}

The GaAs/AlAs SL sample used in this work is grown by molecular beam epitaxy. It consists of 30 periods of $14 \mathrm{~nm}$ GaAs well and $4 \mathrm{~nm}$ AlAs barrier and is sandwiched between two $\mathrm{n}^{+}-\mathrm{GaAs}$ layers. The central $10 \mathrm{~nm}$ of each GaAs well is doped with $\mathrm{Si}$ $\left(\mathrm{n}=2 \times 10^{17} \mathrm{~cm}^{-3}\right)$. The sample is fabricated into $0.2 \times 0.2 \mathrm{~mm}^{2}$ mesas. The sample temperature is fixed at $97 \mathrm{~K}$, at which the temperature-induced formation of DVB is observed in the I-V. For clarity, only part of I$\mathrm{V}$ from the first sequential tunneling plateau is given in Fig.1. It shows the presence of one DVB, along with two complete saw-tooth-like branches. The SSCOs from this DVB is given in the inset of Fig.1 with dc bias at $537 \mathrm{mV}$ and the SSCO frequency $f_{0}$ at 2.89 KHz.

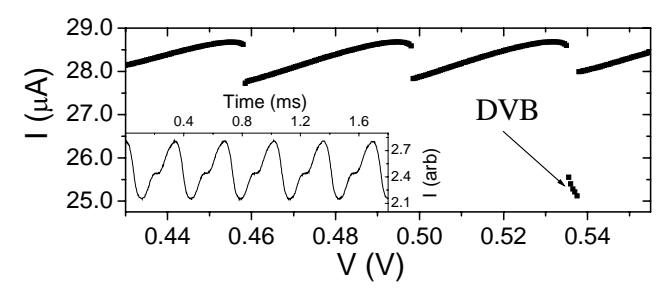

FIGURE 1. I-V characteristics show the coexistence of SVB and DVB. Inset: SSCOs obtained in the DVB indicated.

The influence of a small external ac bias on the I-V characteristics is investigated with a fixed ac frequency $(3 \mathrm{KHz})$ but varying amplitude $\mathrm{V}_{\mathrm{ac}}(0-$ $14 \mathrm{mV}$ ). Figure 2 shows the obtained results in greyscale plot. The measured time-average current is 
shown in density plot where darker area corresponds to lower current value. For clarity, the dc bias range in Fig.2 is the same as in Fig.1. Referring to Fig.1 it is clear that the dark area in Fig. 2 corresponds to DVB, where the current is smaller than that at SVB. In Fig.2, two interesting features are observed. One is that the DVB is induced at the first and second saw-tooth-like branches when $\mathrm{V}_{\text {ac }}$ is larger than about $2 \mathrm{mV}$. The other is the expansion of each DVB with increasing $\mathrm{V}_{\mathrm{ac}}$. At $\mathrm{V}_{\mathrm{ac}}=12 \mathrm{mV}$, the three DVBs join up together turning the whole dc voltage region into dynamic one.

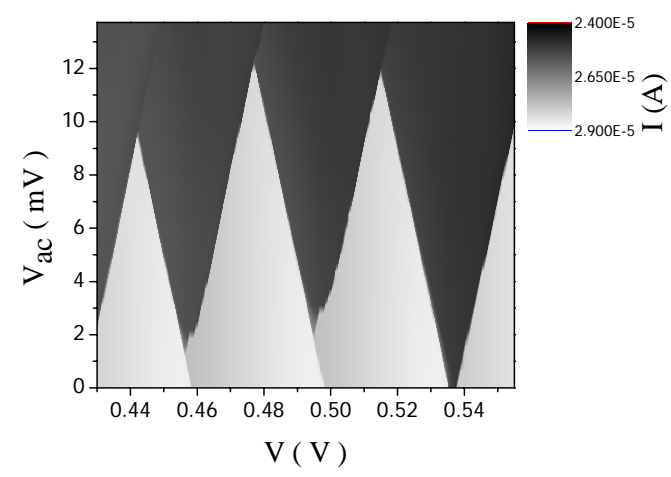

FIGURE 2. The grey scale plot showing the influence of an ac signal amplitude on the DVB.

\section{DISCUSSTION}

In our previous work, it has been shown that the transport dynamics of weakly coupled SL can be well described in the framework of nonlinear dynamics. The saw-tooth like I-V characteristics corresponds to a stable fixed point (SFP) in phase space (see Fig.3(c)), but the formation of a stable limit cycle (SLC) (see Fig.3(a)) results in the SSCO [7]. Based on these, we propose in the intermediate region of the control

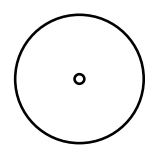

(a)

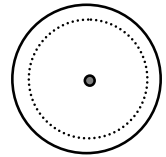

(b) (c)
FIGURE 3. (a)-(c) show the phase portraits corresponding to the three different regions of control parameter space.

parameter space the phase portrait of the system consists of a SLC (solid circle), an unstable limit cycle (ULC) (dot circle) and a SFP (solid dot) as illustrated in Fig. 3 (b). In this region, when the control parameters $\left(\mathrm{N}_{\mathrm{D}}, \mathrm{T}, \mathrm{B}, \mathrm{V}\right)$ are varied in the direction to bring the system from SLC to SFP, the SLC and ULC are forced to get closer and closer. At a critical value, the two limit cycles collide and annihilate leaving only a SFP in the phase space (see Fig.3 (c)). On the other hand, when the control parameters are varied to bring the system from SFP to SLC, the ULC shrinks and finally swallows the SFP, rendering it unstable (see the open dot in Fig.3(a)). As a result, a SLC becomes the only stable solution of the system. To explain our experimental results, the influence of an external ac bias added in the intermediate region of control parameters can be considered as to separate the SLC and ULC and force ULC to tighten on the SFP. As $V_{\text {ac }}$ increases and reaches a critical value the ULC annihilates the SFP turning it into an unstable fixed point. As a result, the system changes from SFP to SLC solution and the SSCO is observed. However, the critical value of $\mathrm{V}_{\mathrm{ac}}$ depends on the other control parameters. In this experiment, only is applied dc bias $\mathrm{V}$ varied. Different V corresponds to different critical values of $\mathrm{V}_{\mathrm{ac}}$. Within a saw-tooth-like current branch, the critical $\mathrm{V}_{\mathrm{ac}}$ increases with increasing V. As applied $\mathrm{V}_{\mathrm{ac}}$ is gradually increased the DVB emerging first at the lower voltage side in the branch is becoming wider and wider. So as shown in Fig.2, the inducing of DVB at the first and second branches and the expansion of the DVB formed at the third branch is observed.

In conclusion, the influence of an ac bias on the emergence and expansion of DVB is studied experimentally. It reveals that the width of DVB increases with the ac amplitude and finally the whole plateau exhibits SSCOs. The underlying mechanism is given based on the general nonlinear analysis of phase portraits in phase space.

\section{ACKNOWLEDGMENTS}

This work is supported by UGC, Hong Kong, through grants HKUST6149/00P and HKUST6162/0P.

\section{REFERENCES}

* corresponding author email: phjwang@ust.hk

1. L. Esaki et al., IBM J.Res.Develop. 14, 61 (1970); R. Tsu et al., Appl.Phys.Lett. 22,562 (1973) ; L. Esaki et al., Phys.Rev.Lett. 33, 495 (1974).

2. K. K. Choi et al., Phys. Rev. B. 38, 12362 (1986) ; H. T. Grahn et al., Phys. Rev. Lett. 67, 1618 (1991); P. Helgesen et al., J. Appl. Phys. 69, 2689 (1991); Y. Zhang et al., Appl. Phys. Lett. 64, 3416 (1994); S. H. Kwok et al., Phys. Rev. B. 50, 2007 (1994).

3. S. H. Kwok et al., Phys. Rev. B. 51, 10171 (1995) ; J. Kastrup et al., Phys. Rev. B. 52, 13761 (1995) ; H. T. Grahn et al., Jpn. J. Appl. Phys. 34, 4526 (1995).

4. J. N. Wang et al., Appl. Phys. Lett. 75, 2620 (1999) ; X. R. Wang et al., Phys. Rev. B. 61, 7261 (2000) ; J. N. Wang et al., Solid State Commun. 112, 371 (1999).

5. Y. Zhang et al., Phys. Rev. Lett. 77, 3001 (1996).

6. J. Kastrup et al., Phys. Rev. B. 55, 2476 (1997); A. Wacker et al., Phys. Rev. B. 55, 2466 (1997).

7. Z. Z. Sun et al., Phys. Rev. B. 69, 045315 (2004). 